\title{
IONS: A Quadruped Robot for Multi-terrain Applications
}

\author{
Sharda Prasad Agrawal, Haresh Dagale, Nirmal Mohan, and L. Umanand
}

\begin{abstract}
In this paper we present the design and development of the IONS (Indoor Outdoor Navigation and Surveillance), a versatile, low-cost, power autonomous, statically stable quadruped robot. The robot has unique compliant wheeled-leg limb design that enables it to climb up and descend stairs of various riser heights. It is designed for statically stable walking, turning, climbing and descending gaits using minimal terrain sensing and simple clock driven open-loop control mechanism. It is equally effective in indoor as well as outdoor settings. Its salient features include auto-alignment of the body while climbing stairs, distinction between stairs and obstacles, collision avoidance etc. The robot is controlled using a web based user interface under ubiquitous Wi-Fi network. The operator use live video feed streamed from on-board camera mounted on the robot platform for gait regulation. On-board system keeps track of signal strength, detects communication failure and sends health status report of the robot to the operator console at regular intervals.
\end{abstract}

Index Terms-Quadruped, stair climbing, compliant leg mechanism, navigation and surveillance, gait regulation, autonomous robot, leg synchronization.

\section{INTRODUCTION}

Many applications that include search and rescue, surveillance and security, require information about an unexplored terrain that may be potentially hazardous for humans. To explore such places we need mobile autonomous robots with multi-terrain traversing capability. Robotic platforms suitable for these applications can be wheeled, legged [1] or one of the many other types [2] in between. Wheeled robots are very efficient on smooth and continuous surfaces but suffer serious limitations in rough terrain and discontinuous paths. On the contrary, legged robots provides superior mobility in natural terrain [3]. They adapt to surface irregularities by using discrete footholds and making contact with ground at the selected points. However, the construction and the control mechanism of a legged robot is complex compared to a wheeled robot. This is mainly because legged robots have multiple actuators and multiple degrees of freedom per leg for better control.

In addition to the wheeled and the legged robots discussed above, there exist a category that falls in between. We refer to them as whegged (wheeled-legged) robots. They generally employ multi-spoked leg design. The complexity of the multi-spoked whegged robots falls in between wheeled and legged [2]-[4] robots. The design of whegged robots generally limits degrees of freedom per leg to 1 or 2. Whegged robots have advantages of both, rough terrain mobility of the legged

Manuscript received November 12, 2014; revised June 2, 2015.

Sharda Prasad Agrawal, Haresh Dagale, Nirmal Mohan and L. Umanand are with Indian Institute of Science, Bangalore, India (e-mail: \{sharda.p, haresh,mnirmal\}@cedt.iisc.ernet.in). robots, as well as simpler control of the wheeled robots. Further, the whegged robot designed with more than two spokes per hip is statically stable [2].

Our objective is to develop a low cost solution for exploration and monitoring the area in and around the multistory building in urban and suburban settings. We have followed whegged design approach for the locomotion of IONS robot. It can be categorized among those robots that can negotiate stairs with riser height greater than their hip height [2], [5]. The 3D Solidworks model and the implemented prototype of the IONS robot are shown in the Fig. 1(a) and Fig. 1 (b) respectively.

The paper is organized as follows, in the next section we provide a brief overview of the related work. In System Design we present mechanical, electrical and software design of the robot. It also discusses various control strategies and communication aspects. Some empirical results of the implemented prototype are discussed in Experimental Results. Finally, we conclude this paper by discussing some possible advancements in the future.

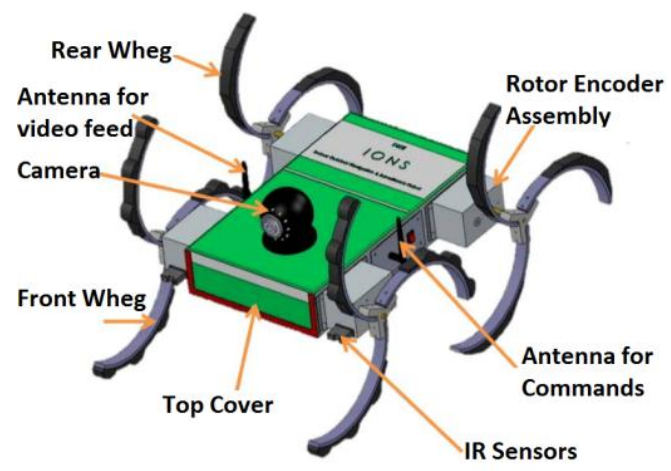

(a) The 3D model

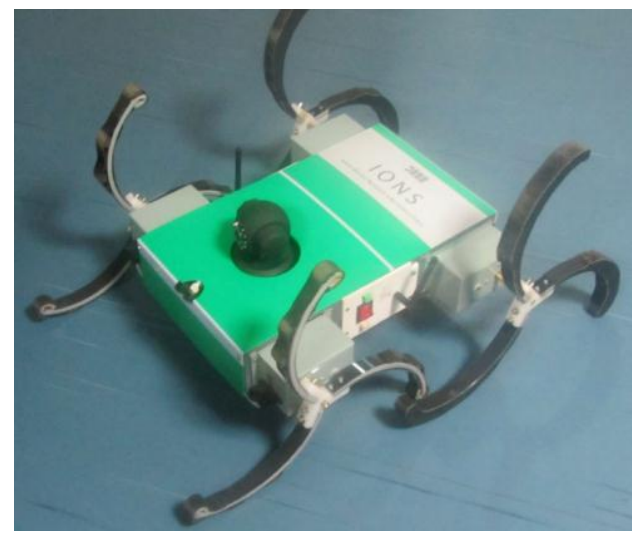

(b) The implemented prototype.

Fig. 1. The IONS robot.

\section{RELATED WORKS}

The survey of rich body of work done in this area has prompted us to classify existing robot designs depending on 
their core functionality.

\section{A. Closed Loop Gait Planning Designs}

This category consists of robotic platforms that uses elaborate sensing elements to estimate the terrain type and use adaptive algorithms to plan its route, gait and speed [6]. RiSE [7] robot is one of the example for this category. They use closed loop gait planning mechanism. These are generally computationally intensive and rich in terms of number of sensors used. For example, RiSE use joint position sensors, leg strain sensors and foot contact sensors in each one of its six legs.

\section{B. Open Loop Gait Planning Designs}

This category consists of robotic platforms that uses clever mechanical structures to minimize the complexity and the cost. They use simple, open loop gait planning mechanism. The leg motions are periodic but have clever built-in compliant mechanism that helps in adapting to the irregularity of the terrain [8]. Loper [4], R-Hex [5] and Whegs [2] robots are the examples for this category. These robots can achieve impressive results on the terrain for which they are designed. However, they perform poorly in the situations where careful footstep planning or significant terrain adaptation is required.

After considering the above discussed design types, it is clear that the closed loop gait planning designs are better from the performance point of view. However, these are complex and tends to be expensive solutions. We found that there are not many low cost, small sized robots that can satisfy both the application requirements, i.e. efficiently negotiating rough terrain and indoor locomotion. Further, our targeted application does not require elaborate sensing and route planning mechanism. Hence, we use the design philosophy embraced by the second category, i.e. open-loop gait planning mechanism with compliant mechanical structure.

\section{SYSTEM DESIGN}

The targeted application scenario makes it essential to have the following features in the robot:

- Sufficient endurance to complete the task

- Traverse efficiently in indoor/outdoor settings and negotiate stairs of various riser heights

- Support control from a safe remote location and send alarm signals in case of failures

The above feature are discussed in the subsequent subsections.

\section{A. Mechanical Design}

The mechanical design of the IONS robot is implemented with the help of its 3D model (Fig. 1(a)) designed using SolidWorks tool. It allowed us to study various mechanical properties of the robot, such as aesthetics, dimensions, component placement, etc before physical implementation. The Fig. 1(b) shows the prototype that is built in the department workshop.

The IONS robot consists of four compliant whegs attached to a semi-complaint rectangular metallic chassis in a similar fashion as Loper and Wheg robots. The chassis is made of Aluminum, whereas the top cover is made of polycarbonate sheet. The chassis has the dimension of $32 \times 37 \times 8 \mathrm{~cm}$ $(\mathrm{W} \times \mathrm{L} \times \mathrm{H})$.

\section{B. Wheg Design}

The robot has three curved leaves in each wheg. The curved leaves have been used in other robots like R-Hex [9], [8]. They are preferred over straight spokes as it allows a greater component of the ground reaction force in the vertical direction [5]. The robot attains static stability, if the number of leaves per wheg are greater than two. However, increasing the number of leaves while keeping the radius same reduces the type of stairs that it can negotiate. Also, it offers much less control over the ground reaction forces [2]. The Fig. 2(b) shows the comparison between a spoked wheg and a curved wheg in the process of a climbing step. Two positions, pos 1 and pos 2 are shown in the figure. In the curved wheg, the torque requirement for pos 1 and pos 2 is $\left(F^{*} D 12\right)$ and $\left(F^{*} D 22\right)$ respectively. Whereas, in the spoked wheg, the torque requirement for pos 1 and pos 2 is $\left(F^{*} D 11\right)$ and $\left(F^{*} D 12\right)$ respectively. Here, $F$ is the vertical load on the hip joint of the wheg, $D 11, D 12, D 21$ and $D 22$ are the positional distances between the hip joint and the point of contact on the surface. It is imperative from the figure that, for the similar position, the distances in the curved wheg are smaller than the spoked wheg due to the curvature of the spoke. Moreover, material property of the spoke further increases the curvature of the wheg when the additional force due to payload comes into play. Thus, the effective length between the hip joint of the wheg and the point of contact on the surface decreases and that reduces the torque required from the motor. Further, this arrangement also reduces the current consumption of the motor and that in turn improves the endurance of the robot.

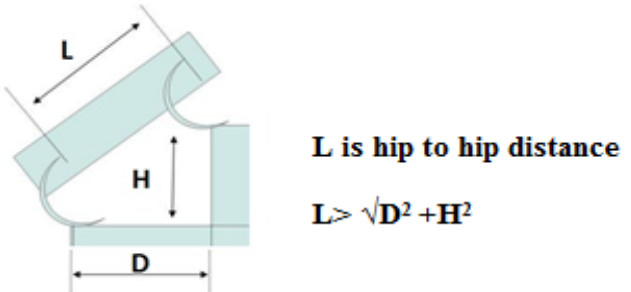

(a) Robot chassis size calculation

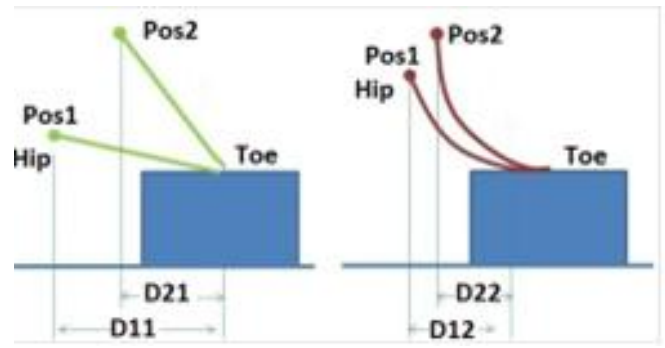

(b) Curved leaf v/s straight spoke.

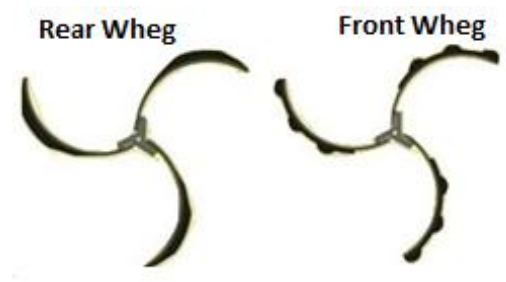

(c) The front and rear wheg.

Fig. 2. The mechanical design of IONS. 
The curved leaves are made using high density elastic plastic material. The elastic steel plates are affixed on the top and the bottom of the curved leaves to provide strength. This arrangement prevents distortion of the curvature of the leaf under stress condition. Curved leaves are connected using a central delrin piece as shown in the Fig. 2(c). The front and the rear whegs have the diameter of $21 \mathrm{~cm}$ and $23 \mathrm{~cm}$ respectively. The dimensions of the wheg and the chassis are calculated based on the targeted applications. For example, the Fig. 2(a) shows the typical design calculation requirement for the chassis length. Various parameters like the targeted stair height, dimensions of the whegs, dimensions of the motors used, maximum track width, and turning radius were taken into account for deciding the size of chassis and wheg diameter. Also, the track width of the front whegs is kept smaller than that of the rear whegs. As a result, the length of the chassis is smaller than the sum of the diameters of the front and the rear whegs. This helps robot to take turn more efficiently and run smoothly.

The front and the rear whegs of the IONS robot are shown in the Fig. 2(c). The front wheg have smaller diameter compared to the rear wheg. This arrangement helps to keep the center of gravity of the robot inside the robot's body triangle while climbing stairs. Also, the front whegs and rear whegs have different padding profile as shown in the Fig. 2(c). The different padding profiles helps in controlled climbing and hence, avoids potential slips. The front whegs have rubber padding with small equidistant humps that provides firm gripping with the surface while climbing. The rear whegs have trapezoidal padding profile that ensures smooth traversing trajectory. The profile and the positions of the hump are derived from the empirical results.

\section{Electronic Hardware and Software Design}

A microcontroller based system is used as the central controller in the robot. The important components of the system are shown in the block diagram given in the Fig. 3(a). The robot has four $12 \mathrm{~V}$ DC motors that drives each wheg independently. For this purpose, we have used the geared DC motors that provides $32 \mathrm{~kg}-\mathrm{cm}$ stall torque. The absolute position of each wheg is measured by a magnetic encode assembly that consist of diametric magnet and AS5040 magnetic encoder. The encoder provides 10 bit positional output that provides $0.35^{\circ}$ resolution. The magnetic encoder assembly use 1:1 gear set that transfers the wheg position to the magnetic assembly as shown in the Fig. 3(b). The magnetic encoder output is supplied as the current position to the central PD controller. The controller computes the next wheg position depending on the command sequence from the operator interface.

The robot has following additional sensors:

- Current sensor - to measure current drawn by the robot from the battery

- Accelerometer - to detect the slop of the terrain

- IR sensors - for obstacle detection and for indoor navigation

As shown in the Fig. 3(a), the robot uses STM32F4 ARM Cortex M4 Discovery board as a central controller platform that runs Chibi OS, a real-time operating system, on it. It uses SPI (Serial Peripheral Interface), PWM (Pulse Width
Modulation), ADC (Analog to Digital Converter) interfaces to provide additional functionality using external devices and controller. Rechargeable, 3 cell $(12 \mathrm{~V})$ Li-Po battery that provides $5000 \mathrm{mAh}$ current rating is used as a central power source for the robot. Two power domains are isolated using DC-DC converter that gives isolation of $4 \mathrm{KV}$ and converts $12 \mathrm{~V}$ to $5 \mathrm{~V}$. STM32F4 board, UART-WI-Fi module, magnetic encoder, IR sensors and IP camera use this isolated power supply of $5 \mathrm{~V}$. Whereas, motor controller use non isolated $12 \mathrm{~V}$ power supply for driving the motors. Further, the signals from the controller board to the motor driver have been isolated using opto-isolator. Isolated power supply is used to avoid any ground loop and EMI issues.

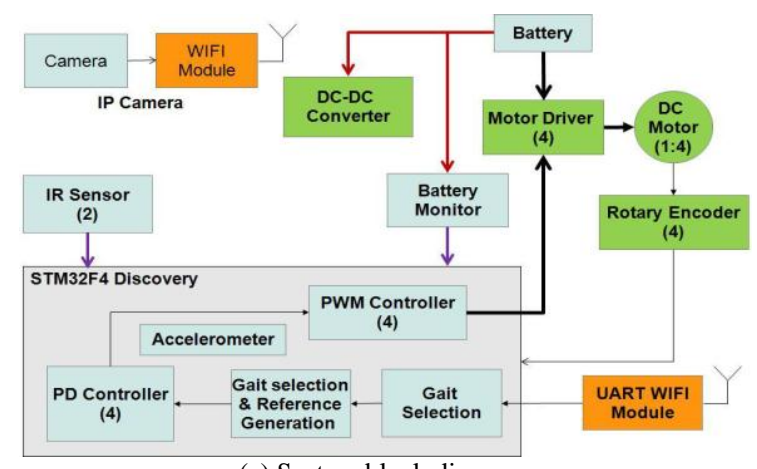

(a) System block diagram

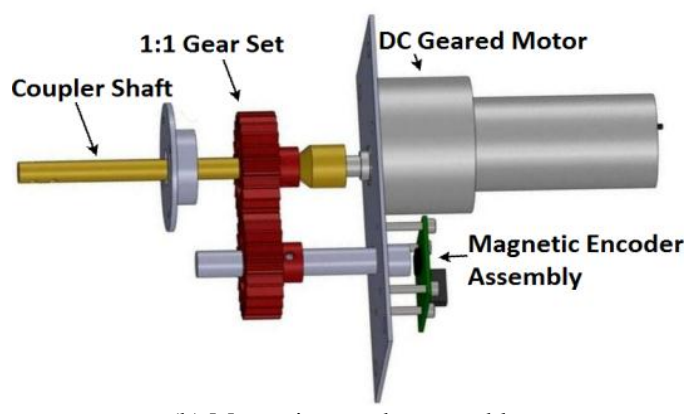

(b) Magnetic encoder assembly.

Fig. 3. System block diagram and magnetic encoder assembly.

\section{Control Strategy}

The absolute position of each wheg is important in a whegged robot. To ensure the position of each wheg with minimum error and fast response time, a proportional differential (PD) controller is used to control individual wheg. For all the whegs to move in synchronism, a separate controller is used to control each wheg. PD control is useful for fast response controllers that does not require zero steady-state error. The "P" action provides an instantaneous response to the error signal that improves the response time of a stable system. The "D" action acts on the rate of change of the control error, which provides a fast response but cannot correct steady state errors. The Integral control drives the system to a steady-state error of zero. The leg position controller does not require the " $I$ " control as there is already an inertia element in the mechanical system that acts as an integrator.

The IONS robot can traverse using different types of gaits that includes walking, turning and stair climbing/descending. These different gaits are achieved by changing the absolute reference to the PD controller. For example, in the left turn, 
the references of the front and the rear whegs on the left side remains constant and for the right side increases linearly with time.

The front and the rear whegs experiences different loads while walking, climbing and descending. During walking and climbing up, the rear whegs experience a higher load because of the gravity and the CoG of the robot. For these gaits, we keep the $\mathrm{P}$ and $\mathrm{D}$ parameters higher for the rear whegs. On the other hand, while climbing down, the front wheg experiences the higher load, hence we keep $\mathrm{P}$ and D parameter higher for the front whegs.

\section{E. Auto Stair Alignment}

The robot has auto align and auto stop features to augment its stair climbing abilities. The auto-align feature provides reliable climbing of stairs irrespective of the distance and the angle of approach. The auto alignment algorithm is explained in the Fig. 4(a).

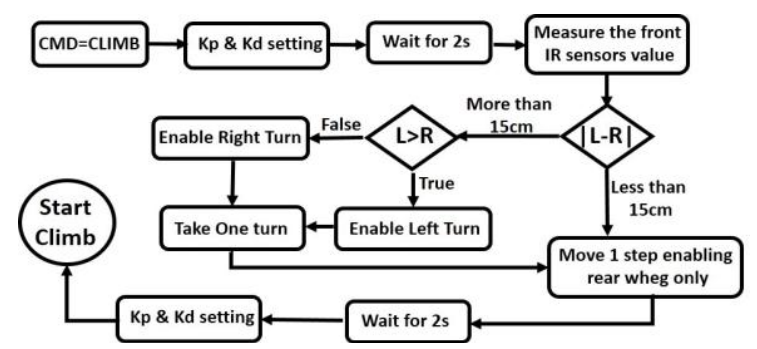

(a) Auto align feature.

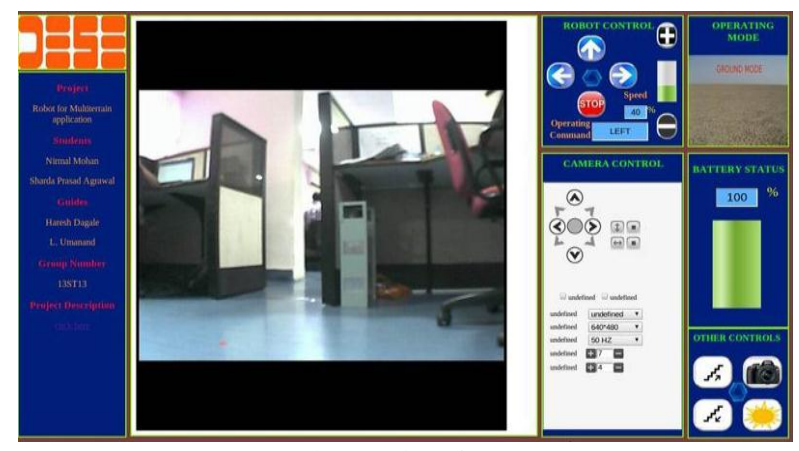

(b) User interface.

Fig. 4. Control Strategies and web interface.

\section{F. Dual Speed Profile}

The linearly increasing reference profile for the PD controller for a wheg, provides a constant speed to the wheg, provided that the load is constant. However, in the real application scenario the wheg experiences a varying load over a complete $360^{\circ}$ rotation. For example, wheg leaf experiences higher load when it makes contact with the ground, otherwise it experiences no ground reaction force. We implemented a dual speed profile to solve this problem. This provides better control over linearly increasing speed profile. The whegs rotates at two different speeds at different times over one full $360^{\circ}$ rotation. The motor rotates slowly when the wheg leaf makes contact with the ground, otherwise it rotates fast. With Dual speed profile we are able to achieve better speed control, better load management and smooth wheg trajectory.

\section{G. User Interface and Command Processing}

A web based user interface is provided for controlling and monitoring purpose. The web page displays the live video feed streamed from the IP camera mounted on the robot platform. The screen shot of the webpage is shown in the Fig. 4(b). The user interface (UI) enables the operator to navigate the robot and adjust pan and tilt of the camera. It also displays the type of terrain it is negotiating and various health parameters of the robot. The Web Server sends various commands to the robot in the form of packets over Wi-Fi network. The command is extracted from the received packet. A state machine is used to guard against spurious commands that may be received by the robot. The extracted command is then processed by the main controller in the robot. Also, the robot periodically sends back health status report to the operator.

\section{EXPERIMENTAL RESULTS}

We have performed several experiments to justify real life usability of our robot and to analyze its performance for stair climbing/descending, normal walking, turning, negotiating obstacles etc. in our department building.

For quantifying the auto alignment feature of the robot, we kept the robot in different positions and the approach angles near the staircase and observed its response. The observations suggests that for the maximum of $45^{\circ}$ approach angle from the normal of the stair case, the robot could auto align itself properly with respect to the staircase. Further, the experiments showed that the endurance of the robot is approx 45 minutes under normal conditions and 28-30 minutes during continuous climbing. It was able to climb one step in two seconds with stable movement.

The experimental results and features of the robot are compared with the existing robots, such as Research RHex and Loper. The results are tabulated in Table I. From the comparisons we can observe that the design and the performance of the IONS is comparable to the existing solutions with the improved leg design.

TABLE I: COMPARISON OF VARIOUS PARAMETER OF DIFFERENT ROBOT

\begin{tabular}{|c|c|c|c|}
\hline \multicolumn{5}{|c|}{ PLATFORM } & $\begin{array}{c}\text { IONS } \\
\text { Robot }\end{array}$ \\
\hline Operating System & QNX & Debian Linux & Chibi OS \\
\hline Total Weight (kg) & $8.2-8.9$ & NA & $6.7-7.0$ \\
\hline $\begin{array}{c}\text { Chassis } \\
\text { L } \times \text { W } \times \text { H(cm) }\end{array}$ & $54 \times 39 \times 12-13.9$ & NA & $42 \times 37 \times 8$ \\
\hline $\begin{array}{c}\text { Ground } \\
\text { Clearance(cm) }\end{array}$ & 11.5 & NA & 8.5 \\
\hline $\begin{array}{c}\text { Front to Rear Leg } \\
\text { Spacing (cm) }\end{array}$ & 20 & NA & 33 \\
\hline $\begin{array}{c}\text { Motor } \\
\text { Type/Weight } \\
\text { (With gearbox)(g) }\end{array}$ & Brushed/292 & AC Servo & Brushed/375 \\
\hline $\begin{array}{c}\text { Gait Regulation } \\
(\text { Hz) }\end{array}$ & 1000 & 400000 & 1000 \\
\hline $\begin{array}{c}\text { Running time } \\
\text { (min) }\end{array}$ & $60-80$ & 40 & $40-50$ \\
\hline $\begin{array}{c}\text { Top speed (m/s) } \\
\text { Range (km) }\end{array}$ & 2.7 & 0.9 & 1.1 \\
\hline
\end{tabular}




\section{CONCLUSION}

The IONS is a low cost robotic platform with unique wheg limbs design. It is designed for civilian applications in urban and suburban settings that includes monitoring and exploration of the area in and around a multistory building. We have compared our prototype with the existing robots reported in the literature. For the targeted application and the cost profile, IONS robot is a simple, light weight robot and performs better in terms of power consumption. The improved power performance is mainly due to clever wheg design. The modular design approach makes it suitable for various application domains. Finally, we conclude this paper by stating that the future advancement will include self-navigation and terrain mapping capabilities in order to make IONS useful in the fire hazardous situations and to act as a fire extinguisher.

\section{REFERENCES}

[1] D. Pongas, M. Mistry, and S. Schaal, "A robust quadruped walking gait for traversing rough terrain," in Proc. 2007 IEEE International Conference on Robotics and Automation, April 2007, pp. 1474-1479.

[2] R. T. Schroer, M. J. Boggess, R. J. Bachmann, R. D. Quinn, and R. E. Ritzmann, "Comparing cockroach and whegs robot body motions," in Proc. 2004 IEEE International Conference on Robotics and Automation, 2004, vol. 4, pp. 3288-3293.

[3] M. F. Silva and J. A. T. Machado, "A historical perspective of legged robots," Journal of Vibration and Control, vol. 13, no. 9-10, pp. 1447-1486, 2007.

[4] S. D. Herbert, A. Drenner, and N. Papanikolopoulos, "A quadruped-hybrid stair climbing robot," in Proc. IEEE International Conference on Robotics and Automation, 2008, pp. 799-804.
[5] U. Saranli, M. Buehler, and D. E. Koditschek, "A simple and highly mobile hexapod robot," International Journal of Robotics Research, vol. 20, pp. 616-631, 2001

[6] P. Manoonpong, F. Pasemann, and F. Worgotter, "Sensor-driven neural control for omnidirectional locomotion and versatile reactive behaviors of walking machines," Robotics and Autonomous Systems, vol. 56, no. 3, pp, 265-288, 2008.

[7] M. J. Spenko, G. C. Haynes, J. A. Saunders, M. R. Cutkosky, A. A. Rizzi, R. J. Full, and D. E. Koditschek, "Biologically inspired climbing with a hexapedal robot," Journal of Field Robotics, vol. 25, no. 4-5, pp. 223-242, Apr. 2008.

[8] K. C. Galloway, G. C. Haynes, B. D. Ilhan, A. M. Johnson, R. Knopf, G. A. Lynch, B. N. Plotnick, M. White, and D. E. Koditschek, "X-rhex: A highly mobile hexapedal robot for sensorimotor tasks," 2010.

[9] U. Saranli, M. Buehler, and D. E. Koditschek, "Design, modeling and preliminary control of a compliant hexapod robot," in Proc. IEEE International Conference on Robotics and Automation, 2000, vol. 3 , pp. 2589-2596.

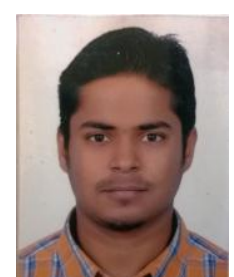

Sharda Prasad Agrawal received his B.E. degree in electronics and communication engineering from RGTU University, India in 2011. He has received his M.Tech degree in electronic systems engineering from Indian Institute of Science, India in 2013. He is interested in robotics locomotion, embedded system design and system integration.

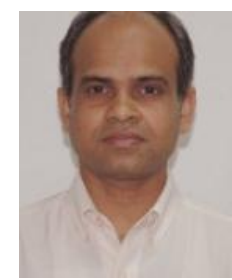

Haresh Dagale is a principal research scientist in the DESE Department, Indian Institute of Science, India He has worked as a scientific officer and the senior scientific officer in the DESE Department. His research area includes object recognition, computer vision, image and video processing. 